Note

\title{
Prediction of the Fungal Response of Fungal Detectors in Dynamic Climate conditions
}

\author{
HIROSHI FUJIKAWA ${ }^{1 *}$, KEIKO ABE $^{2}$, AND SATOSHI MOROZUMI ${ }^{1}$ \\ 'Department of Microbiology, Tokyo Metropolitan Institute of Public Health, 3-24-1 \\ Hyakunin-cho, Sinjuku, Tokyo 169-0073 and 2Institute of Environmental Biology, \\ JDC Corporation, Aikawa-cho, Aikou-gun, Kanagawa 243-0303, Japan
}

Received 14 July 2003/Accepted 26 December 2003

\begin{abstract}
We developed a method to predict the fungal response (FR) of the sensor, Eurotium herbariorum in a fungal detector from the climatic history. Values of $F \boldsymbol{R}$ in dynamic climate conditions were predicted by integrating the rate of $F R$ during the observation periods. FRs predicted under experimental climates were greater than $F R$ s measured, but there was a high linear correlation between them $(r=0.989)$. With an adjustment factor introduced into the method, the increase in the $F \boldsymbol{R}$ value during experimental climates was well predicted. These results suggested that this method might have a potential to accurately predict the $F \boldsymbol{R}$ from the climatic history.
\end{abstract}

Key words : Fungal detector/Fungal index/Growth prediction/Dynamic climate/Fungal growth.

Nowadays people tend to spend more time in airtight houses and buildings with aluminum sashes and/or panels of concrete or synthetic materials. In the moist areas of such airtight places, contaminating molds can easily grow. On the other hand, molds are known to cause allergy in humans (Salvaggio et al., 1981; Verhoeff et al., 1995) and accelerate the growth of mites (Bronswijk and Sinha, 1973; Bronswijk, 1981). Furthermore, mites can also cause allergy in humans (Miyamoto et al., 1968; Shibazaki et al. 1988). Therefore, the control of the growth of mold in houses and buildings has been one of major concerns in public health.

The growth of mold at certain places in buildings and houses is considerably dependent on indoor climates mainly determined by temperature and humidity. Most indoor places such as bathrooms and kitchens supply nutrients for mold to grow. Therefore, indoor climates should be studied for their effect on mold growth. Microclimates in places such as the backs of chests or wardrobes where mold growth is

${ }^{*}$ Corresponding author. Tel : +81-3-3363-3631, Fax : +813-3363-3246. often observed in houses should be also investigated.

Apparatuses such as temperature-humidity recorders are suitable for measuring climates at test places for certain periods. However, the physical parameters measured with these apparatuses have a great essential defect. That is, physical measurements with these apparatuses cannot give direct data about mold growth which we really need. The measured data of climates need to be translated into data on actual mold growth. However, a method for this translation has not been developed so far. Recently Rowan et al. (1999) have analyzed the limits of temperature and relative humidity for mold growth with a polynomial equation. This analysis is not fully quantitative to describe mold growth characteristics, because the limit itself does not represent the kinetics of mold growth.

For quantitative assessment of climates for fungal growth, Abe has recently developed a simple biosensor with a xerophilic mold, Eurotium herbariorum J-183, called a fungal detector (Abe, 1993a, 1993b; Abe et al., 1996). By using the detector, the climatic capacity for fungal growth is estimated as a fungal index. The detector consists of a nutritional source and spores of the sensor mold 
placed between two air-permeable transparent films. The index is evaluated from the fungal growth response, i.e. hyphal elongation, per week under a microclimate in the test place. That is, first, the lengths of elongated hyphae in a fungal detector exposed to a test place for a given period are measured microscopically then transformed to a fungal response, $F R$, to the environment with a standard curve. The index is defined as the value of $F R$ per week (ru /wk), where the response is expressed as response units (ru) (Abe, 2001). The fungal detector has been successfully applied to assess climates in apartments in terms of fungal growth (Abe, 1993a, 1993b; Abe et al., 1996). The detector can also be used in many places such as museums, libraries, and hospitals that must avoid fungal growth (Abe, 1993b).

Under these circumstances, in this study, we newly developed a method to predict the $F R$ of the fungal detectors in response to dynamic patterns of temperature and $\mathrm{RH}$. Then we validated the prediction method under experimental climates.

The fungal index values under various constant climate conditions of temperature and $\mathrm{RH}$ measured previously (Abe, 1993a, 1993b; Abe et al., 1996) were used for the estimation of $F R$. These constant climate conditions ranged from 5 to $35^{\circ} \mathrm{C}$ in temperature and from 71 to $95 \% \mathrm{RH}$. The rate of $F R(\mathrm{ru} / \mathrm{h})$ at

TABLE 1. Experimental climate patterns ( I )

\begin{tabular}{|c|c|c|c|c|c|c|c|c|c|c|c|}
\hline Pattern & Period (h) & $0-24$ & $24-48$ & $48-72$ & $72-96$ & $96-120$ & & & s clima & of cons & $\begin{array}{l}\text { nt cond } \\
\text { neasure }\end{array}$ \\
\hline A & Temp $\left({ }^{\circ} \mathrm{C}\right)$ & 30 & 30 & 30 & 30 & 30 & & were & categoriz & $d$ into 6 & ections \\
\hline & $\mathrm{RH}(\%)$ & 75 & 84 & 92 & 84 & 75 & & & convenie & & \\
\hline & Period $(h)$ & $0-24$ & $24-48$ & $48-72$ & $72-96$ & $96-120$ & & & & & \\
\hline B & Temp $\left({ }^{\circ} \mathrm{C}\right)$ & 20 & 25 & 30 & 25 & 20 & & & & & \\
\hline & $\mathrm{RH}(\%)$ & 95 & 94 & 92 & 94 & 95 & & & & & \\
\hline & Period (h) & $0-24$ & $24-48$ & $48-72$ & $72-96$ & $96-120$ & & & & & \\
\hline C & Temp $\left({ }^{\circ} \mathrm{C}\right)$ & 30 & 25 & 30 & 25 & 30 & & & & & \\
\hline & $\mathrm{RH}(\%)$ & 92 & 84 & 92 & 84 & 92 & & & & & \\
\hline & Period (h) & $0-24$ & $24-48$ & $48-72$ & $72-96$ & $96-120$ & & & & & \\
\hline D & Temp $\left({ }^{\circ} \mathrm{C}\right)$ & 20 & 30 & 25 & 30 & 35 & & & & & \\
\hline & $\mathrm{RH}(\%)$ & 84 & 92 & 85 & 92 & 84 & & & & & \\
\hline & Period (h) & $0-9$ & $9-24$ & $24-33$ & $33-48$ & $48-57$ & $57-72$ & $72-81$ & $81-96$ & $96-105$ & $105-120$ \\
\hline $\mathrm{E}$ & Temp $\left({ }^{\circ} \mathrm{C}\right)$ & 30 & 25 & 30 & 25 & 35 & 25 & 30 & 20 & 30 & 25 \\
\hline & $\mathrm{RH}(\%)$ & 95 & 84 & 92 & 75 & 84 & 94 & 84 & 95 & 84 & 94 \\
\hline & Period (h) & $0-9$ & $9-24$ & $24-33$ & $33-48$ & $48-57$ & $57-72$ & $72-81$ & $81-96$ & $96-105$ & $105-120$ \\
\hline $\mathrm{F}$ & Temp $\left({ }^{\circ} \mathrm{C}\right)$ & 25 & 30 & 35 & 25 & 30 & 25 & 20 & 25 & 30 & 35 \\
\hline & $\mathrm{RH}(\%)$ & 95 & 84 & 92 & 75 & 84 & 94 & 84 & 95 & 84 & 92 \\
\hline
\end{tabular}

Temp is temperature. each climate was estimated by dividing the fungal index value (ru) by the observation period (168 h). The whole profile of the rate is illustrated in Fig. 1.

Predicted FR (PFR) in dynamic temperature- $\mathrm{RH}$ conditions was estimated by integrating the rate of $F R$ with an observation time, as shown in the following equation.

$$
P F R=\int r \mathrm{~d} t \quad[1]
$$

Here $r$ is the rate of $F R(\mathrm{ru} / \mathrm{h})$ at a given temperature and $\mathrm{RH}$, and $t$ is an observation time (h). PFR was calculated by a numerical summation with the spread

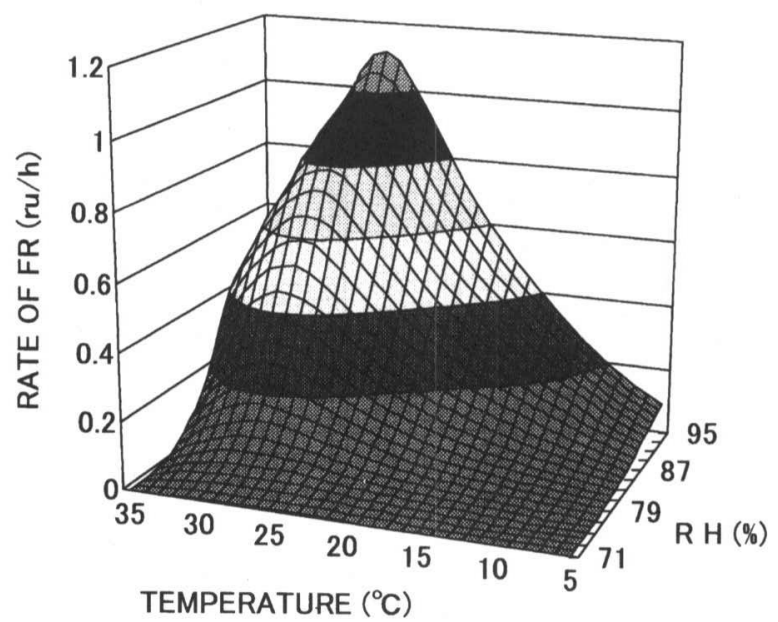

FIG. 1. Fungal response profiles at various climates of constant conditions. Fungal responses measured were categorized into 6 sections for visual convenience. 
sheet calculation software Microsoft Excel.

Fungal detectors with Eurotium herbariorum J-183 (Institute of Environmental Biology, JDC Corporation, Aikawa-cho, Kanagawa, Japan) were placed in duplicate in tightly sealed plastic box chambers $(125 \mathrm{~mm}$ wide, $125 \mathrm{~mm}$ long, $47 \mathrm{~mm}$ high) where RHs were already equilibrated by $40 \mathrm{ml}$ of saturated salt solutions in petri dishes enclosed, for 120 or $240 \mathrm{~h}$. Designated $\mathrm{RHs}$ were made with salts of $\mathrm{NaCl}, \mathrm{KCl}$, and $\mathrm{KNO}_{3}$ (Abe, 1993a). There were no differences between measured values of $\mathrm{FI}$ (or FR) at given $\mathrm{RH}$ s prepared with salt solutions and those prepared with glycerol solutions (Abe, 1993b). A dynamic RH pattern was made by transferring fungal detectors from a chamber equilibrated at a given $\mathrm{RH}$ to another equilibrated chamber. A dynamic temperature pattern was also made by transferring a chamber containing fungal detectors from an incubator (RS-3, Hitachi Co., Tokyo, Japan) equilibrated at a given constant temperature to another chamber equilibrated at a different temperature. After incubation, FRs of the detectors were measured by the method of Abe (Abe, 1993a, 1993b; Abe et al., 1996). Five measurements were done per detector. Out of the five, the average of three interme-

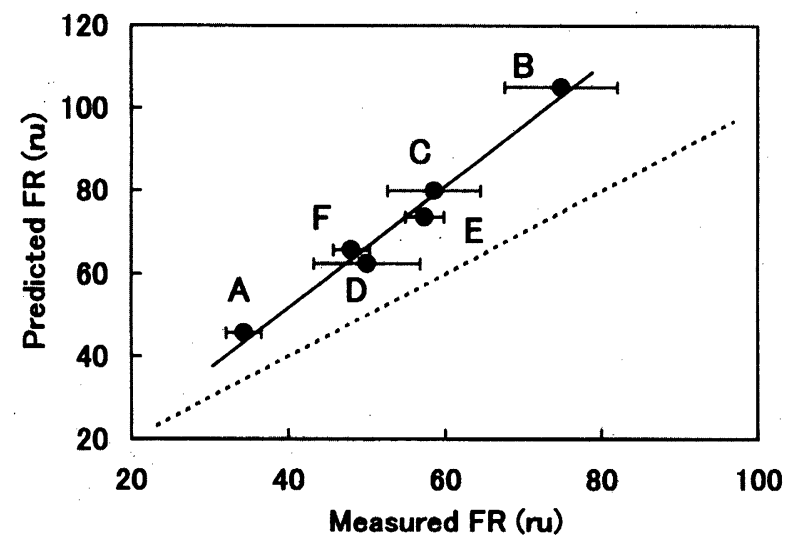

FIG. 2. Linear relationship between measured and predicted FRs in dynamic climate conditions. Alphabets correspond to the climates in Table 1. The straight line is a line of the linear regression. The bar shows the standard deviation of the mean. The dotted line is a line of equivalence. diate values was used for the value of $F R$.

Various types of dynamic climate patterns were tested including a $R H$-varying climate (Table $1 \mathrm{~A}$ ), a temperature-varying climate (Table $1 \mathrm{~B}$ ), and climates with various combinations of temperature and $\mathrm{RH}$ (Table 1C-F). For climate $\mathrm{B}$, the humidity with the $\mathrm{KNO}_{3}$-saturated solution slightly changed (92-95\%) with the temperature. After the exposure to these climates $(120 \mathrm{~h})$, values of measured $F R$ were lower than those of predicted $F R$ (Fig. 2). The average ratio of the measured $F R$ to PFR was $0.75 \pm 0.03$ for the climates tested. However, there was a high linear correlation between them with the correlation coefficient of 0.989 (Fig. 2). This correlation was not much affected by the tested patterns of climate.

These results suggested that equation [1] should be modified by introducing a new factor that adjusts to measured values, as shown in the following equation:

$$
P F R=c \int r \mathrm{~d} t \quad[2]
$$

Here $c$ is an adjustment factor $(0<c \leqq 1)$. The results in Fig. 2 suggested that with an optimal value of $c$, such as 0.75 , FR might be predicted from the climate history using equation [2].

The patterns of measured and predicted FR increases in dynamic climate conditions were then studied. Among the tested climates in Fig. 1, there were several combinations of temperature and $\mathrm{RH}$ with a given value of $F R$ and such climates are located on the contour line of the climatic mountain in the figure. There is a possibility that the rate of $F R$ at a given climate is almost equal to that at climates with different temperatures and/or RHs. In this experiment, therefore, binary climate patterns with different $F R$ rates were studied for evaluation of the modified equation [2] (Table 2). Here, the ratio $(k)$ of the rate of $F R$ under the first climate of the binary change to that under the second one was introduced. Among the combinations of two serial climates, the value of $k$ ranging from 0.91 to 0.10 was tested (Table 2). When the ratio was near one (pattern $A$ ), the increase in $F R$ would be almost linear with time, because the rate of $F R$ in the first climate is almost equal to that in the second. As the ratio was farther

TABLE 2. Experimental climate patterns (II)

\begin{tabular}{|c|c|c|c|c|c|c|}
\hline \multirow{2}{*}{$\begin{array}{l}\text { Pattern } \\
\text { Period }(h)\end{array}$} & \multicolumn{2}{|c|}{ A } & \multicolumn{2}{|c|}{ B } & \multicolumn{2}{|c|}{ C } \\
\hline & $0-9$ & $9-24$ & $0-9$ & $9-24$ & $0-9$ & $9-24$ \\
\hline Temp $\left({ }^{\circ} \mathrm{C}\right)$ & 20 & 30 & 20 & 30 & 25 & 30 \\
\hline $\mathrm{RH}(\%)$ & 85 & 81 & 81 & 85 & 75 & 85 \\
\hline k & \multicolumn{2}{|c|}{0.91} & \multicolumn{2}{|c|}{0.24} & \multicolumn{2}{|c|}{0.10} \\
\hline
\end{tabular}

Fungal detectors were exposed to climates $A$ to $C$ serially with a $24-h$ interval up to (A) $240 \mathrm{~h}$, (B) 120 $\mathrm{h}$, and (C) $120 \mathrm{~h}$. 
from one (patterns $B$ and $C$ ), the pattern of $F R$ increase might be more variable (or zigzag), because of a greater difference between FRs of the first and second climates.

For those climates in Table 2, the modified equation [2] well described the pattern of $F R$ increase (Fig. 3). Here, factor $c$ was determined to minimize the sum of squared differences between the pre-
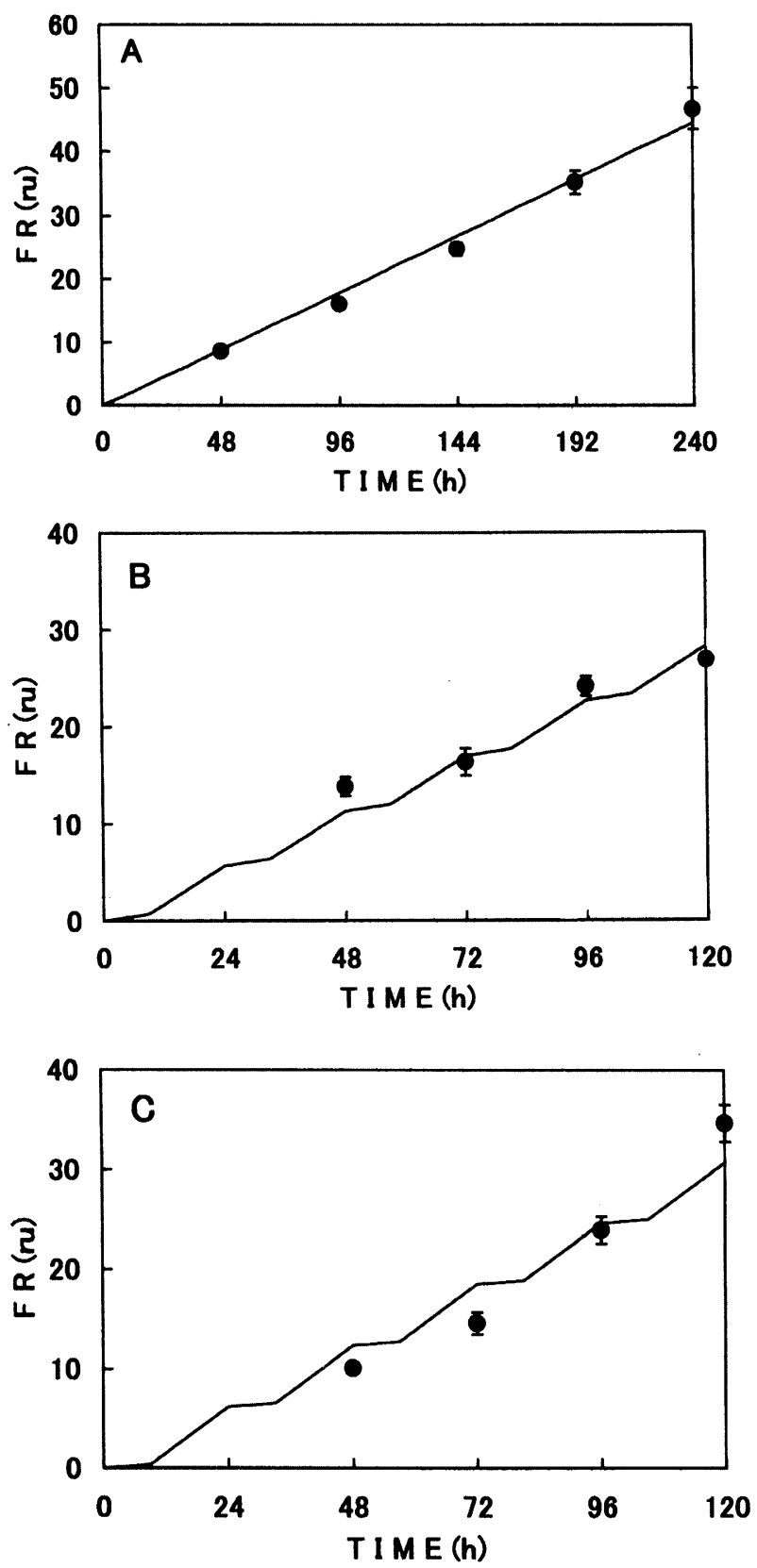

FIG. 3. Kinetics of increasing $F R$ during various types of climates with dynamic conditions. Alphabets correspond to the climates in Table 2. A solid line is a predicted $F R$ and closed circles are measured FRs. The bar shows the standard deviation of the mean. dicted and measured FRs. Values of $c$ were independent of the value of $r$, being (A) 0.71 , (B) 0.63 , and $(C) 0.74$ in Fig. 3, with the average of $0.69 \pm$ 0.057 . As $k$ was smaller, the differences between the predicted and measured $F R$ s were greater; the means of the squared differences between the predicted and measured FRs were (A) 2.4, (B) 2.8, and (C) 9.0.

The kinetics of the increasing $F R$ of the fungal detectors under experimental changing climates was successfully predicted by using equation [2] with an adjusting factor $C$ in this study. However, it has not been clarified why measured FRs were smaller than ones predicted using equation [1] (Fig. 2) and why the difference between the measured FR and the FR predicted with equation [2] was greater with the decrease in $k$ (Fig. 3). Climatic stresses that were brought about by sudden and short-period changes in temperature and/or $\mathrm{RH}$ might suppress the fungal growth of the detector more severely than expected.

As a preliminary finding, we have found that when the period of $\mathrm{RH}<70 \%$ was longer than several hours during the whole observation period, the sensor mold was inactivated and the hyphal elongation stopped (Abe, 1996). Thus, we think that the programming should be modified to stop the calculation in such a case. In the near future, we would like to predict the $F R$ of the fungal detectors under real climates to evaluate the prediction method studied here.

As the $\mathrm{RH}$ of a climate became higher, the growth rate of the sensor mold increased, but at $\mathrm{RH}>95 \%$ the increase in the rate slightly slowed down (Fig. 1). This is because the mold is a xerophilic mold. RHs of the climates in houses, however, are usually lower than $95 \%$, except in special places such as bathrooms. In most indoor climates, thus, the mold in the fungal detector can successfully grow (Abe, 1996). We now think that regardless of using a xerophilic mold as the sensor mold, we can apply the fungal detector to assess mold growth at an indoor place by setting a certain maximum permissible value of $F R$ (or $F /$ ) of the detector as a measure of fungal growth at that place. For indoor climates of places with $\mathrm{RH}>95 \%$, a fungal detector using Alternaria sp., which has been recently found to grow well at such high $\mathrm{RH}$ as well (Abe, 1998), could be utilized.

\section{REFERENCES}

Abe, K. (1993a) A method for numerical characterization of indoor climates by a detector using a xerophilic fungal. Indoor Air, 3, 344-348.

Abe, K. (1993b) A method for the numerical characterization of indoor environment by a detector using a xerophilic fungal. (in Japanese) J. Antibact. Antifung. Agents, 21, 557-565. 
Abe, K., Nagao, Y., Nakada, T., and Sakuma, S. (1996) Assessment of indoor climate in an apartment by use of a fungal index. Appl. Eviron. Microbiol., 62, 959-963.

Abe, K. (1998) Fungal index and contamination in air conditioners when cooled. (in Japanese) J. Soc. Indoor Environ. Jpn., 1, 41-50.

Abe, K. (2001) Fungal index evaluation of indoor environments. (in Japanese) J. Antibact. Antifung. Agents, 29, 557-566.

Bronswijk, J. E. M. and Sinha, R. N. (1973) Role of fungi in the survival of Dermatophgoides (Acarina Pyroglyphidar) in house-dust environment. Environ. Entmol., 2, 142-145.

Bronswijk, J. E. M. (1981) House Dust Biology for Allergists, Acarologists and Mycologosts. NIB Publishers, Zeist. The Netherlands.

Miyamoto, T., Oshima, S., Ishizaki, T. and Sato, S. (1968) Allergic identity between the common floor mite (Dermatophagoides farinae Hughes 1961) and house dust as a causative antigen in bronchial asthma. J. Allergy, 42: 14-28.

Rowan, N. J., Johnstone, C. M., McLean, R. C., Anderson, J. G., and Clarke, J. A. (1999) Prediction of toxigenic fungal growth in buildings by using a novel modelling system. Appl. Environ. Microbiol., 65, 4814-4821.

Salvaggio, J. and Aukrust, L. (1981) Mold-induced asthma. J. Allerg. Clin. Immunol., 68: 327-346.

Shibazaki, M., Tajima, K., Morikawa, A., Mitsuhashi, M., Sumazaki, R., and Tokuyama, K. (1988) Relation between frequency of asthma and $\lg E$ antibody levels against Dermatophagoides farinae and total serum $\operatorname{lgE}$ levels in schoolchildren. J. Allerg. Clin. Immunol., 82, 8694.

Verhoeff, A. P., van Strein, R. T., van Wijnen, J. H. and Brunekreef, B. (1995) Damp housing and childhood respiratory symptoms: the role of sensitization to dust and molds. Am. J. Epidemiol., 141, 103-110. 